\title{
ELASTIC WAVE PROPAGATION FOR CONDITION ASSESSMENT OF STEEL BAR EMBEDDED IN MORTAR
}

\author{
M. RUCKA* and B. ZIMA \\ Department of Structural Mechanics and Bridge Structures \\ Faculty of Civil and Environmental Engineering \\ Gdansk University of Technology \\ ul. Narutowicza 11/12, 80-233 Gdańsk, POLAND \\ E-mail: mrucka@pg.gda.pl
}

\begin{abstract}
This study deals with experimental and numerical investigations of elastic wave propagation in steel bars partially embedded in mortar. The bars with different bonding lengths were tested. Two types of damage were considered: damage of the steel bar and damage of the mortar. Longitudinal waves were excited by a piezoelectric actuator and a vibrometer was used to non-contact measurements of velocity signals. Numerical calculations were performed using the finite elements method. As a result, this paper discusses the possibility of condition assessment in bars embedded in mortar by means of elastic waves.
\end{abstract}

Key words: elastic wave propagation, embedded bar, non-destructive testing, condition assessment.

\section{Introduction}

Rebars embedded in concrete or mortar are commonly used as constructional elements in civil engineering applications. The inserts in the form of bars, wires, strings, cables and nets can be used as the reinforcement. Reinforced concrete structures like tall buildings, bridges, silos or dams are made of a composite material with steel bars placed in concrete. Other types of structures reinforced with bars or wires are ground anchors and rock bolts. Ground anchors are intended for supporting retaining walls while rock bolts are widely used for reinforcement of rocks and tunnels. They consist of cement grouted, prestressed tendons installed in soils or rocks (Sabatini et al., 1999; Wang et al., 2009; Szulborski et al., 2013). Despite relatively high durability of reinforced concrete structures, they are subjected to continuous degradation due to excessive loads and atmospheric conditions. The most common problem is progressive deterioration of bond between the steel bar and composite material as well as damage of reinforcement caused by corrosion. Therefore investigation of damage detection methodologies is of great interest to the civil and mechanical engineering community. In recent years various damage diagnostics systems have been developed for the evaluation of reinforced concrete structures (e.g., Hoła and Schabowicz, 2010; Gołaski et al., 2012). Of particular interests are methods based on elastic wave propagation. While guided wave propagation-based techniques have been applied for many years in steel, aluminum and composite structures (e.g., Jurek et al., 2007; Rucka 2010; Żak et al., 2012), currently there is a considerable and growing interest in the use of guided waves for the evaluation of reinforced concrete structures (e.g. Chróscielewski et al., 2012; Rucka and Wilde, 2013) or rock bolt structures (e.g., Beard and Lowe, 2003; Wang et al., 2009; Zou et al., 2010; Rucka, 2013; Rucka and Zima, 2014). The methods based on propagation of ultrasonic waves are also used to assess the degree of corrosion of steel encased in concrete (Ervin et al., 2009, Lu et al., 2013) or detection of locations where delamination occurred at the border of concrete-steel ( $\mathrm{Na}$ et al., 2003).

\footnotetext{
* To whom correspondence should be addressed
} 
This study deals with the experimental and numerical investigations of elastic wave propagation in steel bars partially embedded in mortar. The bars with different bonding lengths were tested. Two types of damage were considered: damage of the steel bar and damage of the mortar. As a result, the paper discusses the possibility of condition assessment of bars embedded in mortar by means of elastic waves.

\section{Wave propagation in bars embedded in mortar}

At the interface between media with different physical properties (i.e., the modulus of elasticity $E$ and the mass density $\rho$ ) two phenomena may occur: a wave can be reflected from the boundary or it can be transmitted into the second medium (Fig.1). Each medium can be characterized by a resistance value called acoustic impedance which is the opposition of a medium to a longitudinal wave motion and is defined as

$$
W=\rho c \approx \sqrt{\rho E}
$$

where $c$ denotes the velocity of the longitudinal wave. The reflection coefficient $R$ and the transmission coefficient $T$ can be written as Rose (1999)

$$
\begin{aligned}
& R=\frac{W_{2}-W_{1}}{W_{1}+W_{2}}=\frac{\rho_{2} c_{2}-\rho_{1} c_{1}}{\rho_{1} c_{1}+\rho_{2} c_{2}}, \\
& T=\frac{2 W_{2}}{W_{1}+W_{2}}=\frac{2 \rho_{2} c_{2}}{\rho_{l} c_{1}+\rho_{2} c_{2}} .
\end{aligned}
$$

The intensity of reflection and transmission depends on the difference between values of acoustic impedance of two media. In the case of two media with different velocity of longitudinal wave propagation, only the part of the wave energy penetrates the second medium, while the remaining part is reflected. A large difference in the impedance can result in complete reflection of a wave, while a small difference in the impedance may be the cause of the dominance of the wave transmission into the surrounding medium. A long, slender bar can be perceived as a waveguide along which so-called guided waves can propagate. Considering the free bar (i.e., the bar surrounded by air), the wave propagates along it and when the wave reaches the bar ends, it is completely reflected. A different situation occurs when the bar is embedded in a material (e.g., mortar) with impedance properties more similar to the impedance properties of steel. In such a case the wave leakage occurs at the interface between the steel and mortar (Fig.2).

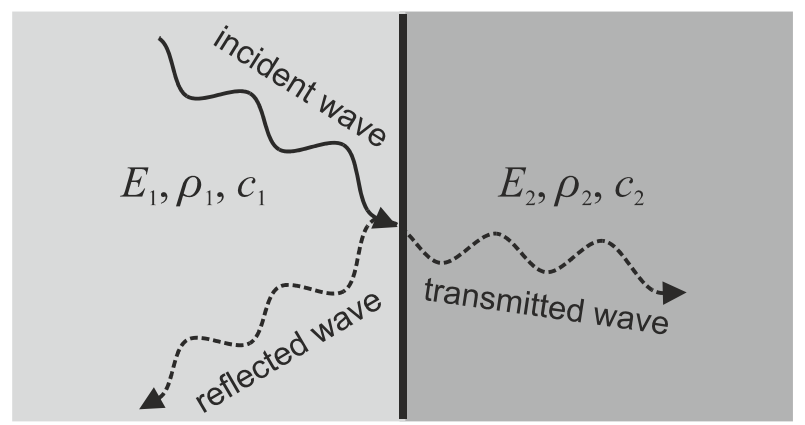

Fig.1. Wave reflection and passing through the boundary between media. 


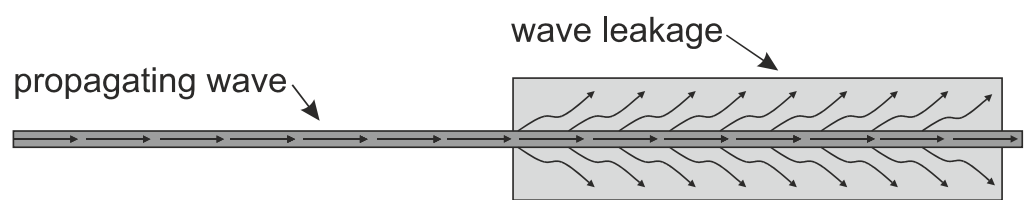

Fig.2. Wave leakage on the steel-mortar interface.

\section{Experimental investigations}

\subsection{Description of specimens}

The specimen tested in this study was a steel bar $\left(E=200 \mathrm{GPa} ; v=0.3 ; \rho=7556 \mathrm{~kg} / \mathrm{m}^{3}\right)$ embedded in $\operatorname{mortar}\left(E=5 \mathrm{GPa} ; v=0.16 ; \rho=1770 \mathrm{~kg} / \mathrm{m}^{3}\right)$. The steel bar has a cross section of $0.6 \times 0.6 \mathrm{~cm}^{2}$ and a length of $1 \mathrm{~m}$ (Fig.3). A defect in the form of a rectangular notch with a depth of $1 \mathrm{~mm}(0.5 \mathrm{~mm}$ on both sides of the bar) and a length of $5 \mathrm{~mm}$ was located at a distance of $67.5 \mathrm{~cm}$ from the left end of the bar. The steel bar was embedded in a rectangular block of mortar with a cross section of $2.6 \times 2.6 \mathrm{~cm}^{2}$ and a length $x$. Figure 3 shows the scheme of the typical tested specimen. During the experiment the mortar cover was gradually removed from the bar length. Consequently, 15 different specimens (of numbers from \#1 to \#15) were made with a mortar length varying from $x=96 \mathrm{~cm}$ (specimen \#1) to $x=0 \mathrm{~cm}$ (specimen \#15). The geometry of all tested specimens is given in Fig.4. Additionally, in some specimens the mortar was destroyed by making a notch with a width of about $5 \mathrm{~mm}$ (Fig.5).

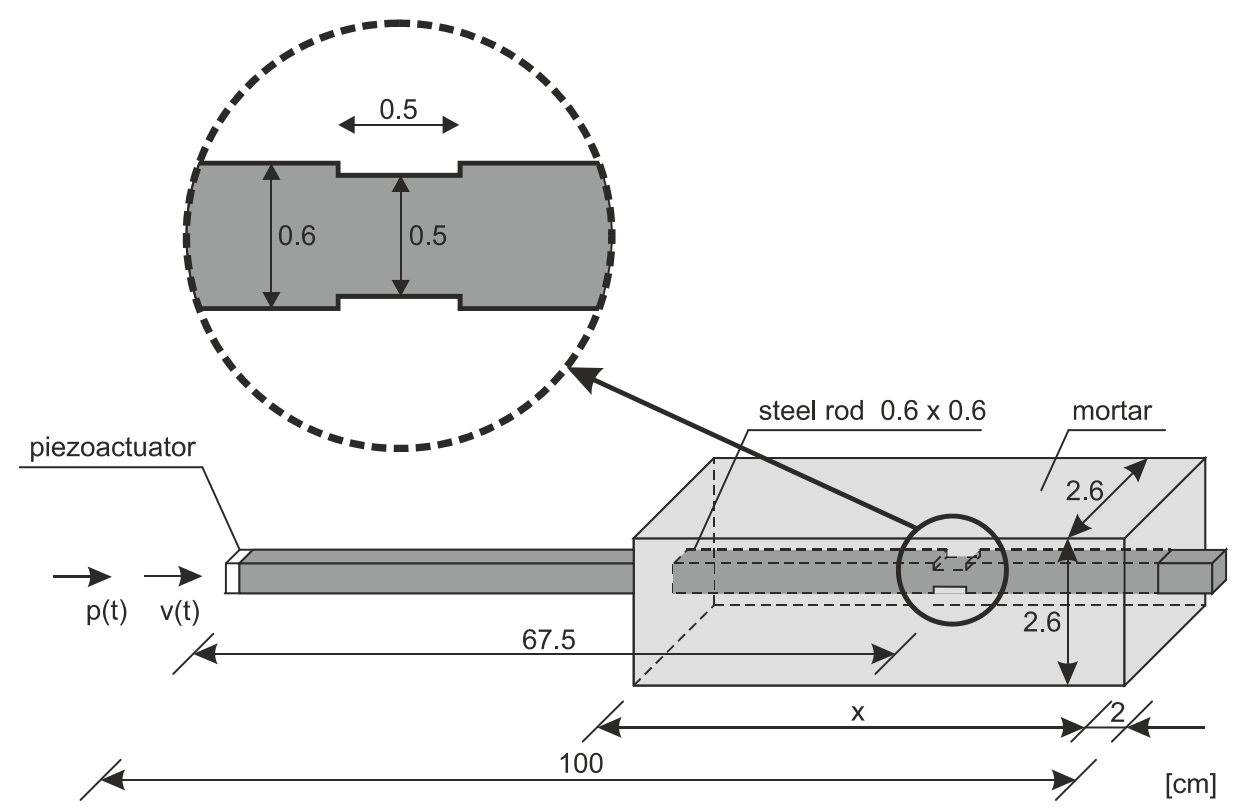

Fig.3. Scheme of the tested specimen. 


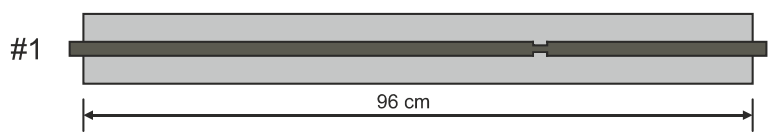

\#9

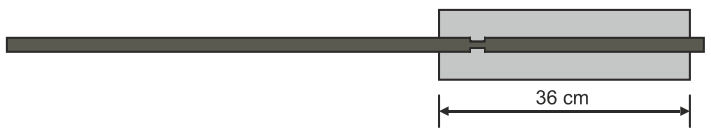

\#2

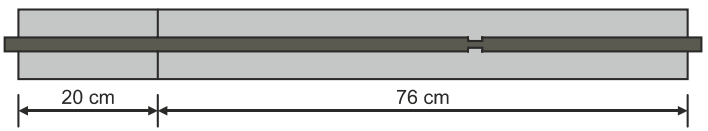

\#10

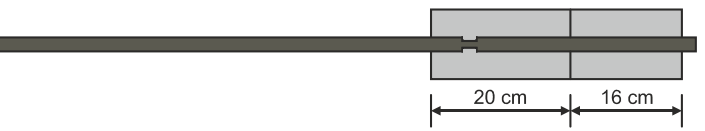

\#3
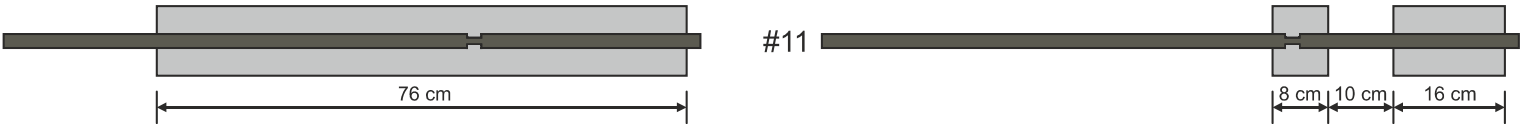

\#4
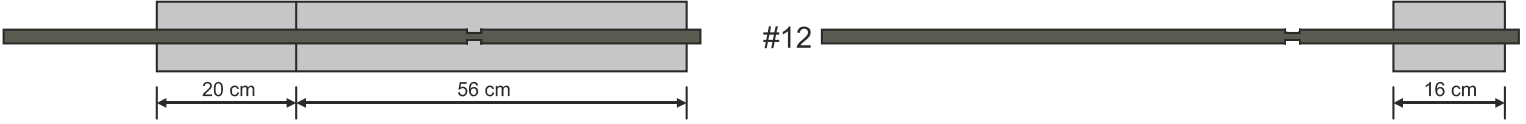

\#5

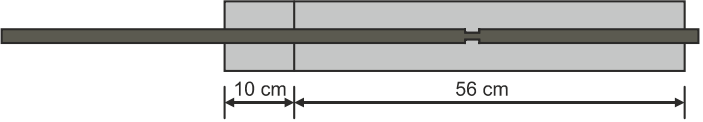

\#6
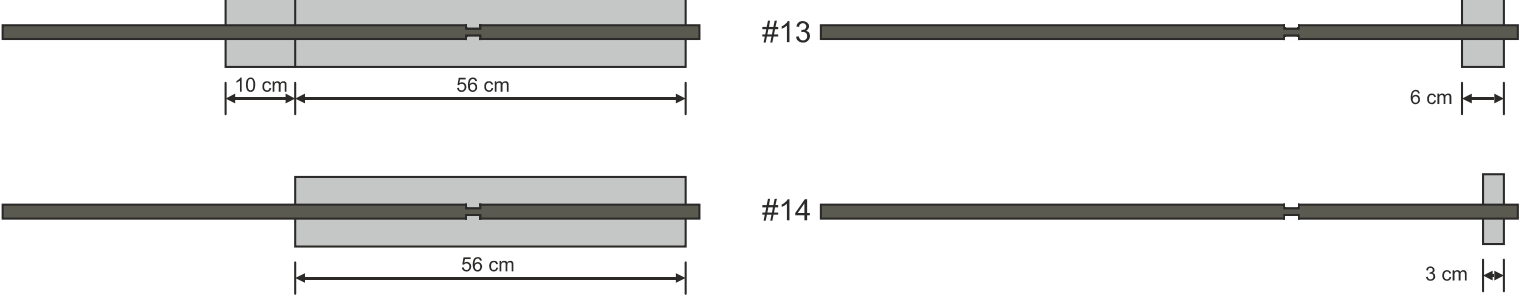

\#7

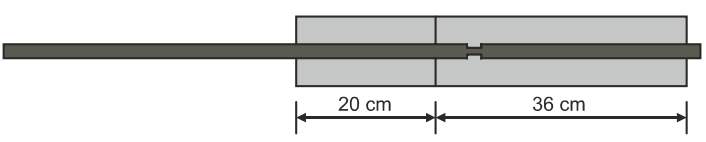

\#15

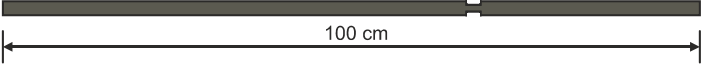

\#8

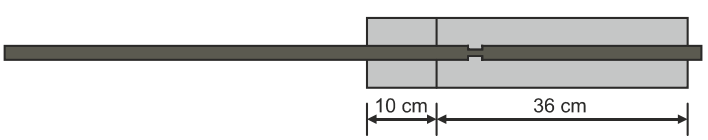

Fig.4. Experimental models (specimens \#1 to \#15) with different bonding lengths.

a)

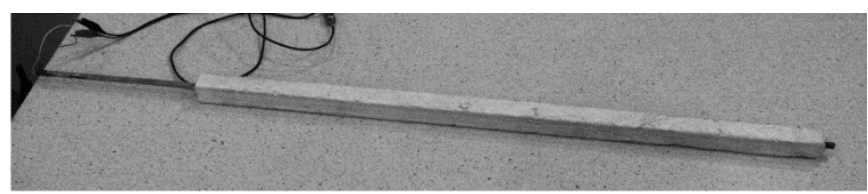

b)

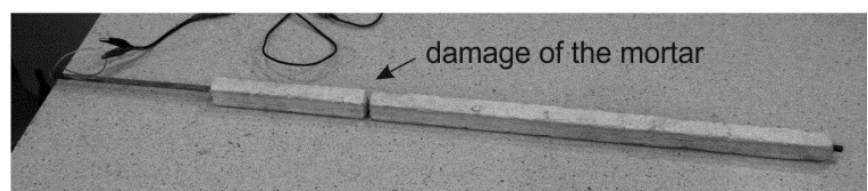

Fig.5. Photographs of bars partially embedded in mortar: a) specimen \#3; b) specimen \#4 (with damage of the mortar).

\subsection{Experimental setup}

The experimental setup for wave propagation is shown in Fig.6. Ultrasonic waves were excited by the arbitrary waveform generator (Tektronix AFG 3022) with the high voltage amplifier (EC Electronics 
PPA 2000). The PZT plate actuator Noliac CMAP11 of dimensions $5 \times 5 \times 2 \mathrm{~mm}^{3}$ was bonded as the actuator on the left end of the bar. Propagating velocity signals $v(t)$ were sensed and recorded by the scanning head of the laser vibrometer Polytec PSV-3D-400-M on the same end where the excitation has been realized (cf. Fig.3). The PZT actuator produced ultrasonic wave which propagated through the specimen in the longitudinal direction. A five-peak wave packet obtained from the multiplication of a sinusoidal function of a carrier frequency $f=100 \mathrm{kHz}$ and a window function $w(t)$ was chosen as the excitation signal $p(t)$

$$
p(t)= \begin{cases}p_{o} \sin (2 \pi f t) \cdot w(t) & t \in\left[0, T_{w}\right], \\ 0 & t>T_{w},\end{cases}
$$

where $T_{w}$ denotes the length of a window and $p_{o}$ is an amplitude of a sinusoidal function. The Hanning window was applied as a window function

$$
w(t)=0.5\left(1-\cos \left(2 \pi f t / n_{w}\right)\right), \quad t \in\left[0, T_{w}\right]
$$

where $n_{w}$ denotes the number of counts in the tone burst.

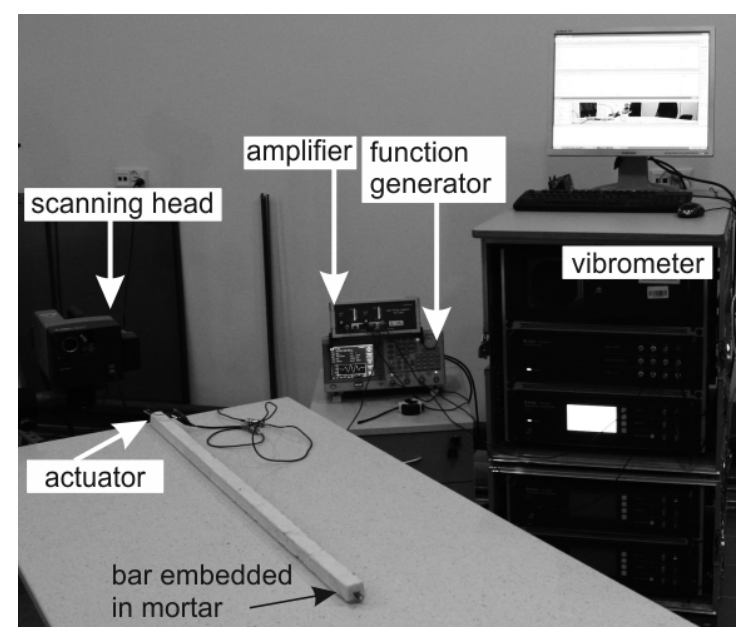

Fig.6. Experimental setup for wave propagation measurements in the bar embedded in mortar.

\section{Numerical simulations of wave propagation by FEM}

Numerical calculations of elastic wave propagation were performed by using the finite elements method in Abaqus/Explicit. The system of the bar embedded in mortar under consideration was discretized using 8-node solid elements (C3D8R) with dimensions $1 \times 1 \times 1 \mathrm{~mm}^{3}$ with reduced integration and hourglass control. The size of the elements was chosen according to the recommendation that more than 20 nodes per wavelength should be used to represent accurately propagating waves (Moser et al., 1999)

$$
l_{e}=\frac{\lambda_{\min }}{20}
$$

where $l_{e}$ is the finite element length and $\lambda_{\min }$ denotes the shortest wavelength. The size of the integration step was set as $\Delta t=10^{-7} \mathrm{~s}$ and it satisfied the rule (Moser et al., 1999) 


$$
\Delta t=\frac{1}{20 f_{\max }}
$$

where $f_{\max }$ is the highest frequency of interest.

The boundary conditions were assumed as free on all edges. The numerical model did not take into account the possibility of worse conditions of adhesion between steel and mortar. Materials of the bar and the cover were assumed to have linear elastic behaviour. The excitation $p(t)$ in the form of the wave packet was realized by the application of a surface load with a value of $30 \mathrm{kPa}$ to the left end of the bar. The velocity signal $v(t)$ was recorded on the same end of the bar (cf. Fig.3).

Figure 7 shows maps of the velocity field propagated in specimen \#6 at selected time instances. After excitation of the wave packet, the guided wave propagated along the free part of the bar (Fig.7a). When the wave reached the anchorage place, a part of the wave was reflected from the anchorage while another part was transmitted. The wave transmitted along the bar leaked into the entire volume of the mortar (Figs $7 \mathrm{~b}, \mathrm{c}$, d) even though only the steel bar was excited.

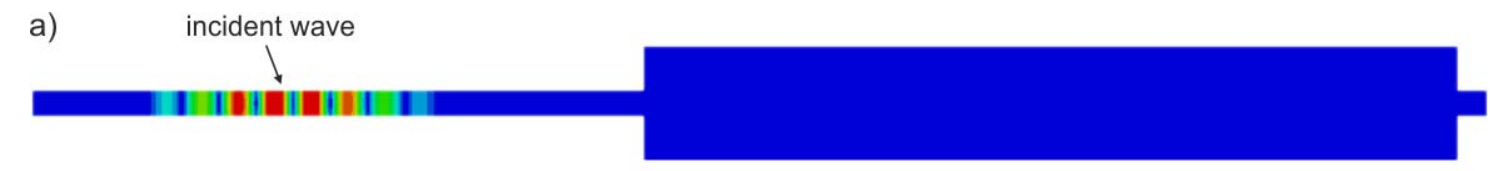

b)

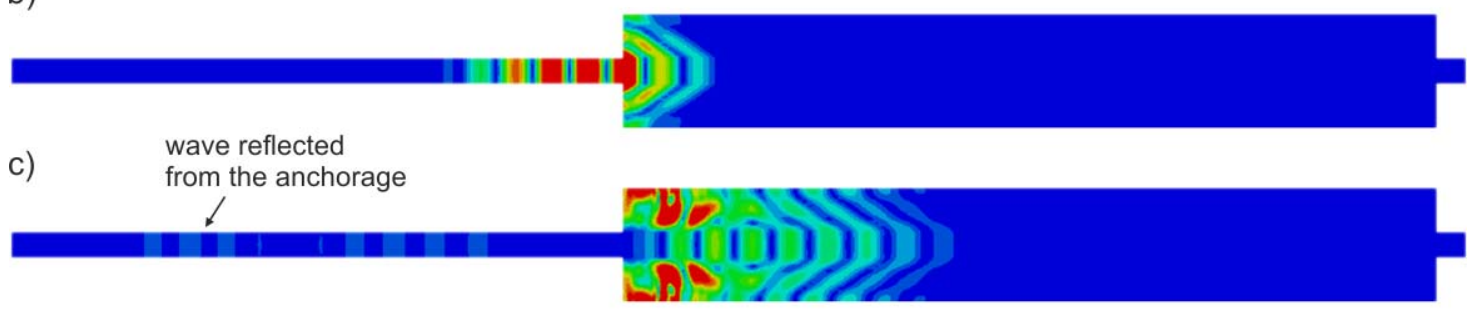

d)

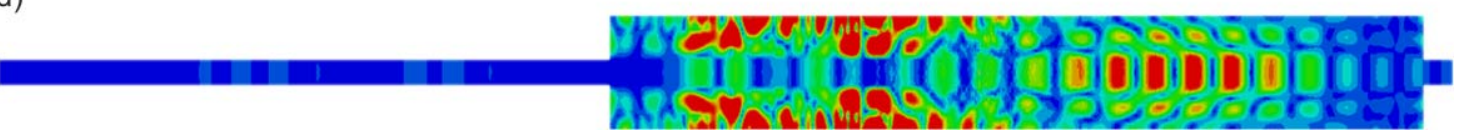

Fig.7. Maps of velocity field propagated in specimen \#6 (component $v_{z}-$ along the axis of the bar) at selected time instances: a) $t=6 \times 10^{-5} \mathrm{~s}$; b) $t=1.05 \times 10^{-4} \mathrm{~s}$; c) $t=1.65 \times 10^{-4} \mathrm{~s}$; d) $t=3.3 \times 10^{-4} \mathrm{~s}$.

\section{Results of wave propagation in bars embedded in mortar}

\subsection{Comparison of numerical and experimental results}

The results of experimental and numerical investigations are presented in Fig. 8 in the form of wave propagation signals recorded at the left end of the bar for all specimens of numbers from \#1 to \#15. In each diagram numerical signals were plotted as envelopes and they were imposed on signals obtained experimentally. To create signal envelopes, the Hilbert transform was implemented according to the formula (Potamianos and Maragos, 1994)

$$
\hat{v}(t)=\frac{1}{\pi} \int_{-\infty}^{\infty} \frac{v(\tau)}{t-\tau} d \tau
$$

where $v(\tau)$ is the registered signal of the propagating wave. 

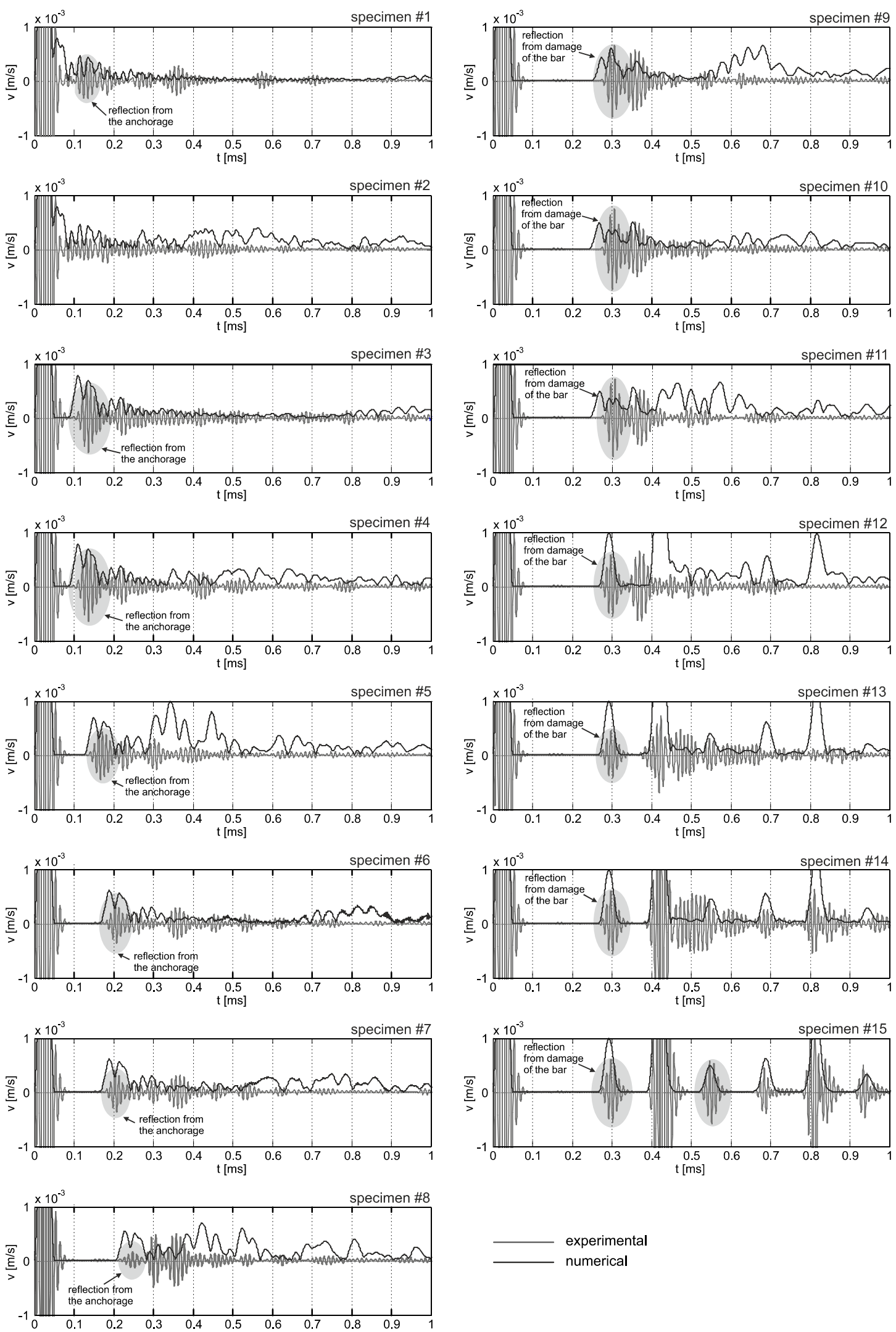

\section{experimental}

numerical

Fig.8. Set of wave propagation signals collected during experiments and corresponding results of numerical investigations (in the form of signal envelopes). 
The compatibility of the experimental and numerical signals can be visible in two ways: reflections from the notch of the steel bar and the anchorage occurred at the same time and the values of the registered amplitudes were consistent. Identification of reflections from the anchorage was more difficult in the cases when the bonding length included almost the whole bar length (Figs 8a, b) because the reflection from the anchorage which is located close to the end of the bar equipped with the actuator became covered with the input wave packet. When the distance between the source of waves and the anchorage was long enough to avoid interference of the excited wave packet and undesirable reflections, localization of the anchorage was easy to identify (see Fig.8) based on the knowledge of the group velocity of the axial wave propagated in the bar and the time of registered reflection in the wave propagation signal.

\subsection{Identification of damage in mortar}

Four pairs of bars embedded in mortar were analyzed. As the reference models, specimens \#1, \#3, \#6 and \#9 were examined. The notch of a width of about $5 \mathrm{~mm}$ was cut in mortar in specimens \#2, \#4, \#7 and $\# 10$ at a distance of $20 \mathrm{~cm}$ from the anchorage (cf. Fig.4, Fig.5). In Fig.9 experimental wave propagation signals for the bars with the same bonding length are presented. The results for bars with damaged mortar are drawn as the signal envelopes. A single measurement for the bar with damaged mortar did not provide information about condition assessment of the structure. Comparing a signal for the bar with damaged mortar with a signal recognized as reference, differences in signal amplitudes can be observed. These small changes, marked with ellipses in Fig.9, can serve as indicators for damage assessment.
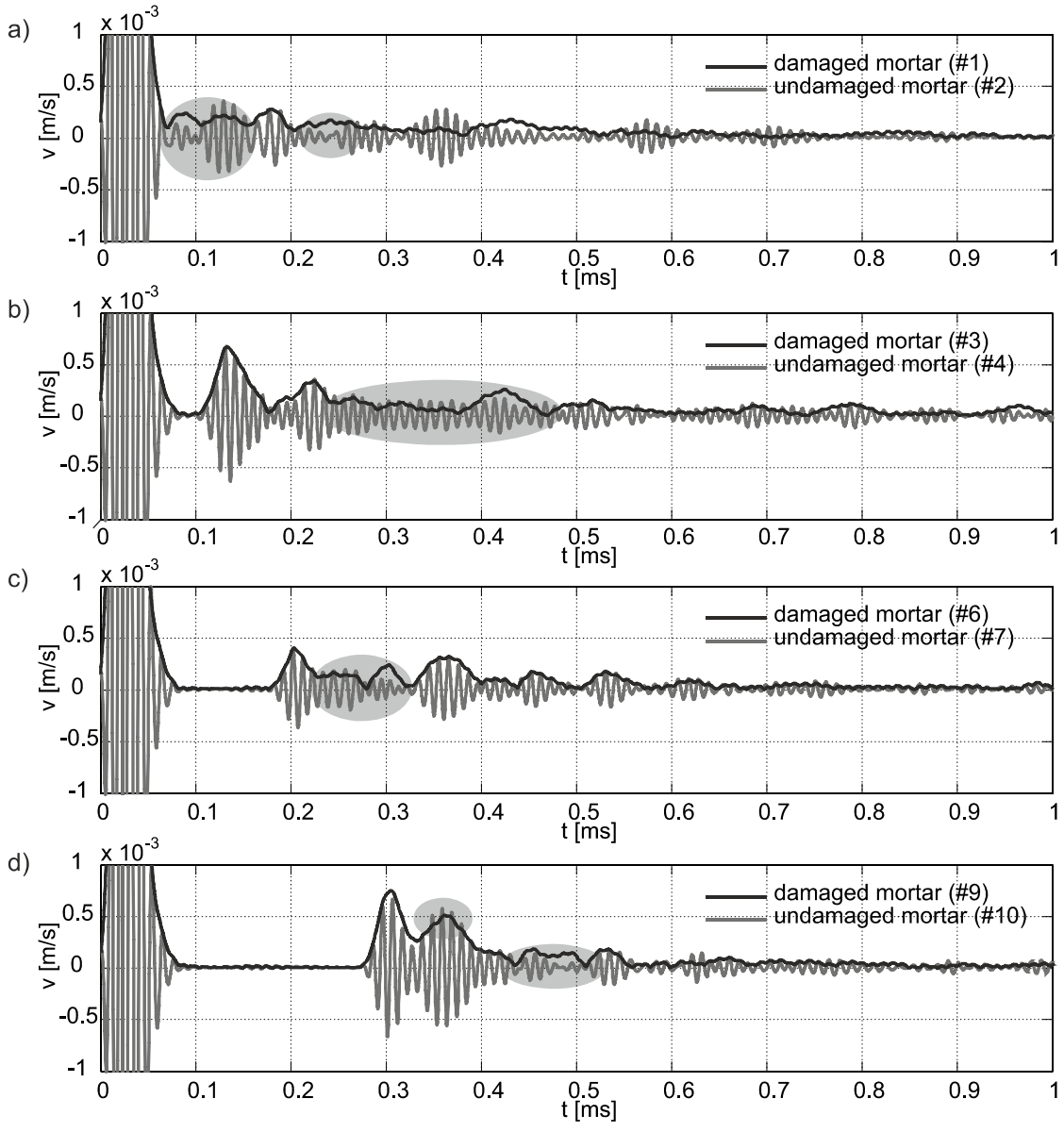

Fig.9. Set of wave propagation signals collected during experiments for bars with the same length of undamaged and damaged mortar: a) specimens \#1 and \#2; b) specimens \#3 and \#4; a) specimens \#6 and \#7; a) specimens \#9 and \#10. 


\subsection{Bar damage identification}

The last stage of investigations concerned the possibility of detection of the notch in the steel bar in the case when damage was covered by mortar. In Fig. 10 envelopes of recorded wave propagation signals for bars with bonding length from $56 \mathrm{~cm}$ (specimen \#6) to $36 \mathrm{~cm}$ (specimen \#10) are presented. For bars with greater bonding lengths than $56 \mathrm{~cm}$ identification of reflection from damage was impossible (cf. Fig.8). Reflection from damage in the bar occurred at the time instance equal to $0.3 \mathrm{~ms}$. As we can see, the amplitude values depend on the bonding length of the bar. Even though the bonding lengths in the case of specimens \#6 and \#7 are practically the same, the amplitude value is different. It is caused by the presence of the notch in mortar. The propagation path along which the leakage occurs is shorter by the width of the notch $(5 \mathrm{~mm})$ and even such a small difference caused changes in registered signals. A similar situation can be observed in the case of specimen \#9 and \#10. For these two bars the distance from the anchorage to damage of the bar is the same because the notch of the mortar has been made behind damage of the steel bar. Therefore the attenuation of guided waves in this case may be caused by worse conditions of adhesion between the steel bar and mortar as a result of cuts during the experiment.
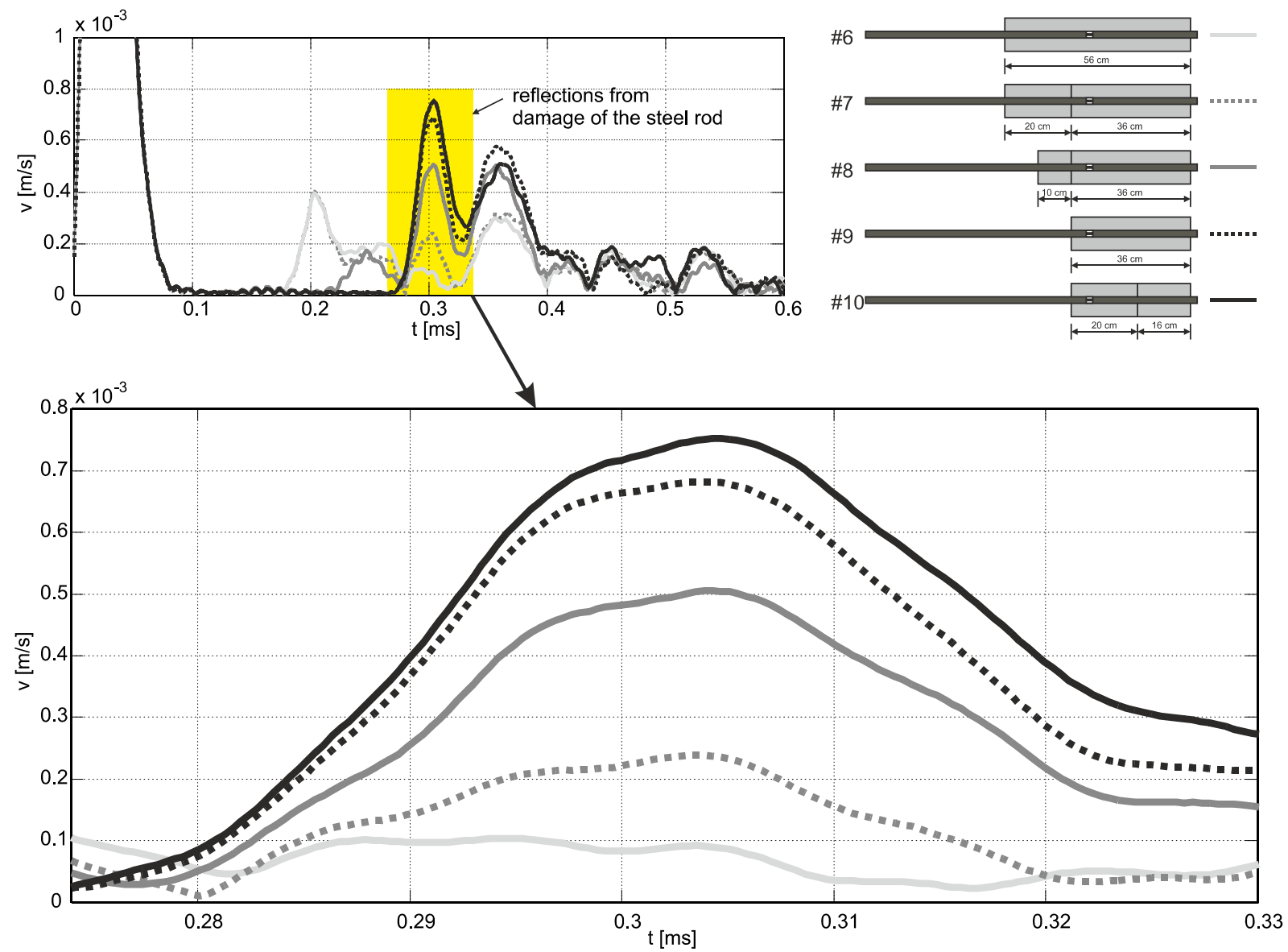

Fig.10. Set of wave propagation signals collected during experiments for bars with different bonding lengths (specimens \#6 to \#10): comparison of amplitudes of reflections from damage of the steel bar.

The attenuation coefficient $K$ is a measure of the loss of the energy transferred into the bar cover (Wang et al., 2009; Paviakovic et al., 2001). In general, it is used to evaluate the grouting quality of the embedded bar. The attenuation coefficient can be expressed by the relation (Wang et al., 2009) 


$$
K=-\frac{20}{2 L} \cdot \log _{10}\left(\frac{A_{2}}{A_{1}}\right)
$$

where $L$ is the length of the bar, $A_{1}$ is the amplitude of the excitation wave and $A_{2}$ is the amplitude of the reflected wave. In this study, the attenuation coefficient was used to assess the energy loss on the base of reflection from damage of the bar. Therefore, length $L$ was equal to $67.5 \mathrm{~cm}$ (the position of the notch) and $A_{2}$ was the amplitude of the wave reflected from this damage. Figure 11 shows the curve of the attenuation coefficient $K$ for specimens of numbers from \#6 to \#10. It can be seen that as the bonding length increases and conditions of adhesion become better, more energy is transferred from the bar into mortar.

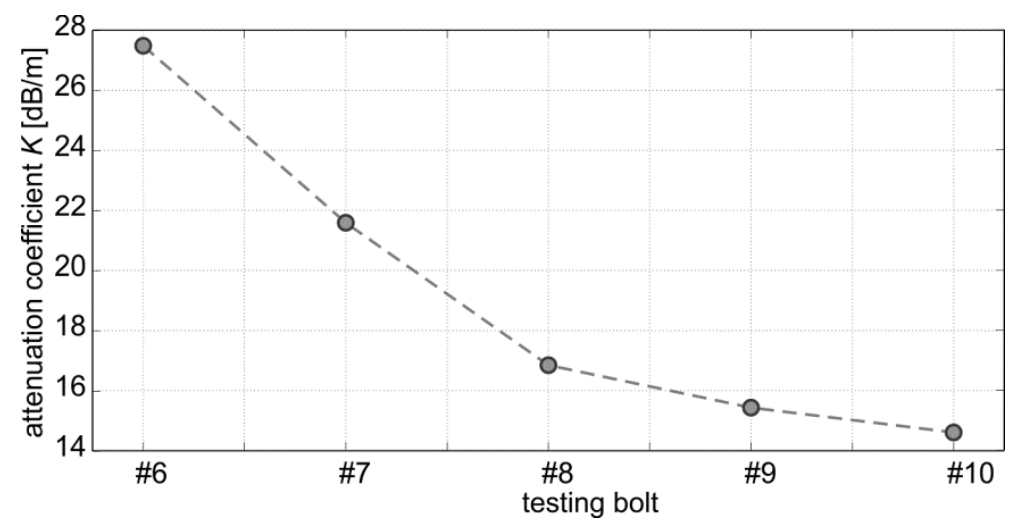

Fig.11. Curve of attenuation coefficient $K$ for specimens $\# 6$ to $\# 10$.

\section{Conclusions}

This paper presents an experimental and numerical study on the possibility of the application of ultrasonic waves for the evaluation of damage in a steel bar embedded in mortar. The specimens with different bonding length were tested. Velocity time histories of elastic waves propagation have been used for condition assessment. Difficulties in extraction and interpretation of information from wave propagation signals resulted from the progressive wave leakage. When the bonding quality of the bar embedded in mortar got better because of the increase of the bonding length, more energy was transferred from the bar into mortar which led to a significant attenuation of the guided wave. It was possible to identify the localization of the anchorage based on the knowledge of the group velocity of the axial wave propagated in the bar and the time or registered reflection in the wave propagation signal.

Two types of damage in the bar embedded in mortar have been considered: damage of the mortar and damage of the steel bar. A single measurement for the bar with damaged mortar did not provide information about condition assessment of the structure. However, a comparison of a signal for the bar with damaged mortar with a signal recognized as reference disclosed differences in signal amplitudes that can be used for assessing the bonding quality. Identification of the notch in the steel bar was possible only in the case when the distance between the anchorage and the defect was smaller than $26 \mathrm{~cm}$. Amplitude values of the wave reflected from the notch cut in the bar varied with the bonding length of the bar. The results proved that with the increase of the bonding length, more energy of the guided wave is transferred into the mortar. For this reason, attenuation of the amplitude of the elastic wave propagating in the anchored steel element can be used to assess the bonding quality of rock bolts. 


\section{Acknowledgements}

Calculations were carried out at the Academic Computer Center in Gdańsk.

\section{Nomenclature}

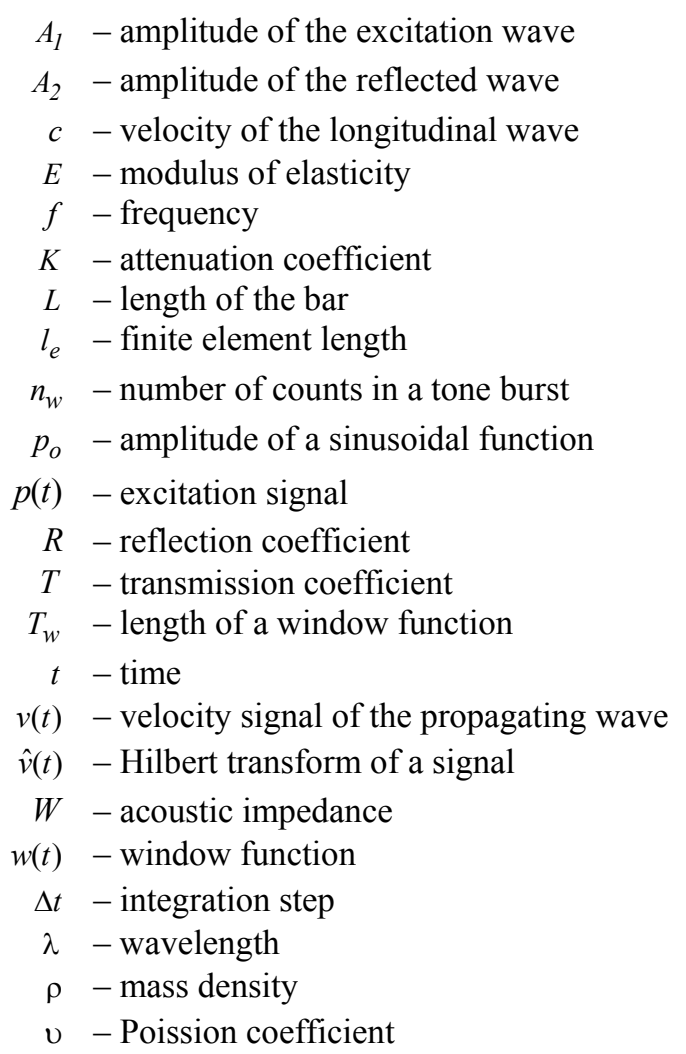

\section{References}

Beard M.D. and Lowe M.J.S. (2003): Non-destructive testing of rock bolts using ultrasonic guided waves. International Journal of Rock Mechanics and Mining Sciences, vol.40, pp.527-536.

Chróścielewski, Rucka M., Wilde K. and Witkowski W. (2012): Diagnostics of concrete beams during bending process using elastic wave propagation (in Polish). - Scientific Letters of Rzeszow University of Technology, No.283, pp.349-356.

Gołaski L., Goszczyńska B., Świt G. and Trąmpczyński W. (2012): System for the global monitoring and evaluation of damage processes developing within concrete structure under service load. - The Baltic Journal of Road and Bridge Engineering, vol.7, No.4, pp.237-245.

Ervin B.L., Kuchma D.A., Bernhard J.T. and Reis H. (2009): Monitoring corrosion of rebar embedded in mortar using high-frequency guided ultrasonic waves. - Journal of Engineering Mechanics, vol.135, pp.9-18.

Hoła J. and Schabowicz K. (2010): State-of-the-art non-destructive methods for diagnostic testing of building structures - anticipated development trends. - Archives of Civil and Mechanical Engineering, vol.10 No.3, pp.5-18.

Jurek M., Nazarko P. and Ziemiański L. (2007): Non-destructive structure testing: structure fault detection based on the elastic waves phenomenon (in Polish). - Scientific Letters of Rzeszow University of Technology, No.243, pp.67-87.

Lu Y., Li J., Ye L. and Wang D. (2013): Guided waves for damage detection in rebar-reinforced concrete beams. Construction and Building Materials, vol.47, pp.370-378. 
Moser F., Jacobs L.J. and Qu J. (1999): Modelling elastic wave propagation in waveguides with the finite element method. - Nondestructive Testing and Evaluation International, vol.32, pp.225-234.

Na W-B., Kundu T. and Ehsani M.R. (2003): Lamb waves for detecting delamination between steel bars and concrete. - Computer-Aided Civil and Infrastructure Engineering, vol.18, pp.58-63.

Potamianos A. and Maragos P. (1994): A comparison of the energy operator and the Hilbert transform approach to signal and speech demodulation. - Signal Processing, vol.37, pp.95-120.

Paviakovic B.N., Lowe M.J.S. and Cawley P. (2001): High frequency low-loss ultrasonic modes in imbedded bars. Journal of Applied Mechanics, vol.68, pp.67-75.

Rose J.L. (1999): Ultrasonic Waves in Solid Media. - Cambridge University Press

Rucka M. (2010): Experimental and numerical studies of guided wave damage detection in bars with structural discontinuities. - Archive of Applied Mechanics, vol.80, pp.1371-1390.

Rucka M. (2013): Contribution to the diagnostics of rock bolts using elastic wave propagation (in Polish). Construction and Architecture, vol.12, No.1, pp.227-234.

Rucka M. and Wilde K. (2013): Experimental study on ultrasonic monitoring of splitting failure in reinforced concrete. - Journal of Nondestructive Evaluation, vol.32, pp.372-383.

Rucka M. and Zima B. (2014): Detection of rebar anchorage length using elastic wave propagation (in Polish). Journal of Civil Engineering, Environment and Architecture, vol.61, pp.257-267.

Sabatini P.J., Pass D.G. and Bachus R.C. (1999): Ground anchors and anchored systems. - Technical Report FHWAIF-99-015, Office of Bridge Technology, Washington.

Szulborski K., Nalewajko R. and Kościńska-Grabowska K. (2013): About the implementation of excavation enclosure formed by diaphragm walls supported with ground anchors in Szczecin (in Polish). - Engineering and Construction, vol.11, pp.575-578.

Wang C., He W., Ning J. and Zhang C. (2009): Propagation properties of guided wave in the anchorage structure of rock bolts. - Journal of Applied Geophysics, vol.69, pp.131-139.

Zou D.H. Steve, Cheng J., Yue R. and Sun X. (2010): Grout quality and its impact on guided ultrasonic waves in grouted rock bolts. - Journal of Applied Geophysics, vol.72, pp.102-106.

Żak A., Radzieński M., Krawczuk M. and Ostachowicz W. (2012): Damage detection strategies based on propagation of guided elastic waves. - Smart Materials and Structures, vol.21, 035024 (18pp).

Received: August 30, 2014

Revised: October 6, 2014 